\title{
An Energy Consumption Model for IEEE 802.11ah WLANs
}

Albert Bel, Toni Adame, and Boris Bellalta

Department of Information and Communication Technologies

Universitat Pompeu Fabra, Barcelona

\begin{abstract}
One of the main challenges when designing a new self-powered Low Power Wide Area Network (LPWAN) technology is the vast operational dependence on its scarce energy resources. Therefore, a thorough identification and characterisation of the main energy consumption processes may lay the foundation for developing novel mechanisms aimed to make a more efficient use of devices' batteries, as well as for planning future network deployments. This paper provides an analytical energy consumption model for IEEE 802.11ah WLANs, which are expected to become one of the technology drivers in the future development of the Internet of Things (IoT). Given the network characteristics, the presented analytical model is able to provide an estimation of the average energy consumed by a station in the network, predicting its battery lifetime, based on a set of closed-form equations. In addition, this model can be used as a tool to understand the effects of the main network parameters on the network energy consumption, and to find the most suitable network configuration for a given application scenario.
\end{abstract}

Keywords: IEEE 802.11ah, WLANs, LPWANs, M2M, WSNs, Power Saving Mechanisms.

\section{Introduction}

The growing use of Machine to Machine (M2M) communications [1] envisages a future where personal and business decision making will be increasingly based on the information provided by unattended systems. Their autonomous, scattered, ubiquitous and non-invasive nature facilitates the procedure of obtaining environmental data from large numbers of sensors, but at the same time supposes a technological challenge, as most of these devices are strongly conditioned by processing, memory and, particularly, energy constraints.

Indeed, neither of the two current IoT/M2M players (cellular networks and Wireless Sensor Networks (WSNs)) has yet been able to create a prevailing technology with such considerations, thus fostering the appearance of new low capability communication standards such as IEEE 802.11ah [2]. Conceived as an amendment of the consolidated and well-known IEEE 802.11 Wireless Local Area Network (WLAN) technology, this amendment offers a competitive long-range solution in the sub $1 \mathrm{GHz}$ band for very large sensor networks (i.e., $>8 \mathrm{~K}$ devices) with low power consumption and short-burst data transmission requirements 
( $<100$ Bytes). To achieve this, besides modifying the IEEE 802.11ac PHY layer to operate in the sub 1 $\mathrm{GHz}$ band, IEEE 802.11ah includes new power management mechanisms [3, 4,5]. One of them, called TIM and page segmentation [6], extends the IEEE 802.11 power saving mechanism [7] and distributes network stations and channel resources according to a novel hierarchical method. Essentially, energy consumption of an station (STA) is reduced by limiting the number of possible contenders in its corresponding TDMA-like transmission period.

Analytical characterization of IEEE 802.11ah WLANs has been already considered by the research community. In [8], an analytical model to characterize the performance in terms of throughput of the TIM and page segmentation scheme is proposed, although no calculations of energy consumption are included. On the contrary, [9] surveys the performance (collision probability, delay, and battery lifétime) of IEEE 802.11ah networks with periodic traffic, while [10] predicts their saturation throughput and energy efficiency assuming known collision and error probabilities. As for IEEE 802.11ah simulations,/a model to calculate the maximum number of stations using power saving mechanisms is presented in [11], a performance assessment of its power saving mechanism is included in [12], and a novel low-consuming channel access mechanism is proposed in [13].

This paper presents an analytical model for the energy consumption in an IEEE 802.11ah WLAN, where all elements of the TIM and page segmentation scheme/(including signalling beacons, number of stations per group, transmission periods, and so forth) were taken into consideration. In addition, the model accuracy was evaluated by comparing its predictions with the results presented in [12], where the energy consumption of an IEEE 802.11ah WLAN in four typical M2M scenarios (agriculture monitoring, smart metering, industrial automation and animal monitoring) was obtained by means of an 'ad-hoc' built simulator. The high similarity between the proposed model and the simulations proves the model effectiveness and quickness when predicting the energy consumption and the average lifetime of an IEEE 802.11ah WLAN. Moreover, once the model was validated, it was used to study the suitability of different IEEE 802.11ah configurations in terms of energy consumption and packet reliability.

The remainder of this paper is organised as follows: Section 2 provides the main parameters and assumptions of the considered IEEE 802.11ah network, and Section 3 details the equations of our analytical model. Its performance is evaluated in Section 4, and the evaluation of suitable network configurations is provided in Section 5. Lastly, Section 6 presents the conclusions and discusses open challenges.

\section{System Model}

\subsection{IEEE 802.11ah WLAN operation}

IEEE 802.11ah extends the IEEE 802.11 power saving mechanism (PSM) [7] by using a scheme called TIM and page segmentation, which reduces the time an STA is competing for the channel and increases its 
sleeping periods.

IEEE 802.11ah introduces a novel hierarchical method to build groups of stations depending on an association identifier. This hierarchical distribution of stations into groups, called TIM groups, is used not only for organizational purposes but also for signalling and allocating available channel resources, allowing stations to enter into a sleep mode during non-traffic periods. Hence, an STA only wakes up at predefined moments to listen to the following beacons:

1. DTIM (Delivery Traffic Indication Map) beacons. They must be listened to by all STAs, as they are used to signal which TIM groups have pending data (unicast and/or multicast) in the AP. DTIM beacons are broadcast from the AP every $T$ seconds.

2. TIM (Traffic Indication Map) beacons. They page a single TIM group with at least one station having pending data in the AP. Between two DTIM beacons, there are as many TIM beacons as TIM groups $\left(N_{\mathrm{TIM}}\right)$.

After listening to a DTIM beacon and, occasionally to a multicast (MC) data transmission, an STA with pending downlink (DL) and/or uplink (UL) data will wake up to listen to its corresponding TIM beacon. It includes information about the subsequent restricted access window (RAW), where different $\psi \in\{\mathrm{DL}, \mathrm{UL}, \mathrm{MC}\}$ transmission procedures can be performed.

As shown in Figure 1, RAW windows consist of one downlink segment $\left(\mathrm{RAW}_{\mathrm{DL}}\right)$, one uplink segment $\left(\mathrm{RAW}_{\mathrm{UL}}\right)$ and, for the first window after a DTIM beacon, one multicast segment $\left(\mathrm{RAW}_{\mathrm{MC}}\right)$. Hence, an STA with data to transmit or receive is only awake during its corresponding $\mathrm{RAW}_{\psi}$ segment, remaining in sleeping mode otherwise. $\mathrm{RAW}_{\psi}$ segments must be long enough to include all time periods involved in a $\psi$ transmission procedure: the ones corresponding to data $\left(T_{\mathrm{DATA}}\right)$ as well as control $\left(T_{c t r l}\right)$ frames (namely, PS-POLL, ACK, RTS, and CTS frames). The duration of these frames will depend on their length $\left(L_{\text {DATA }}\right.$ and $\left.L_{c t r l}\right)$ and the selected data rate $(R)$, being $T_{\mathrm{DATA}}=\frac{L_{\mathrm{DATA}}}{R}$ and $T_{c t r l}=\frac{L_{c t r l}}{R}$.

In this paper, where only STAs using the TIM and page segmentation scheme have been considered, the channel access combines an AP-centralized time period allocation system with the distributed coordination function (DCF) medium-access technique within those periods. DL/UL transmission procedures are shown in Figure 1 and detailed as follows:

1. Downlink: When an STA has one or more data packets pending to receive, it will be first informed by the inclusion of its TIM group in the DTIM bitmap, and later by its own inclusion in the TIM bitmap. To initiate the packet reception, the STA will send a PS-POLL frame in its allocated RAW ${ }_{\text {DL }}$ segment.

2. Uplink: Whenever an STA wants to send a UL message to the AP, it must first wait for its corresponding $\mathrm{RAW}_{\mathrm{UL}}$ segment. Once within it, the STA will start the data transmission by using basic access or RTS/CTS mechanism. 


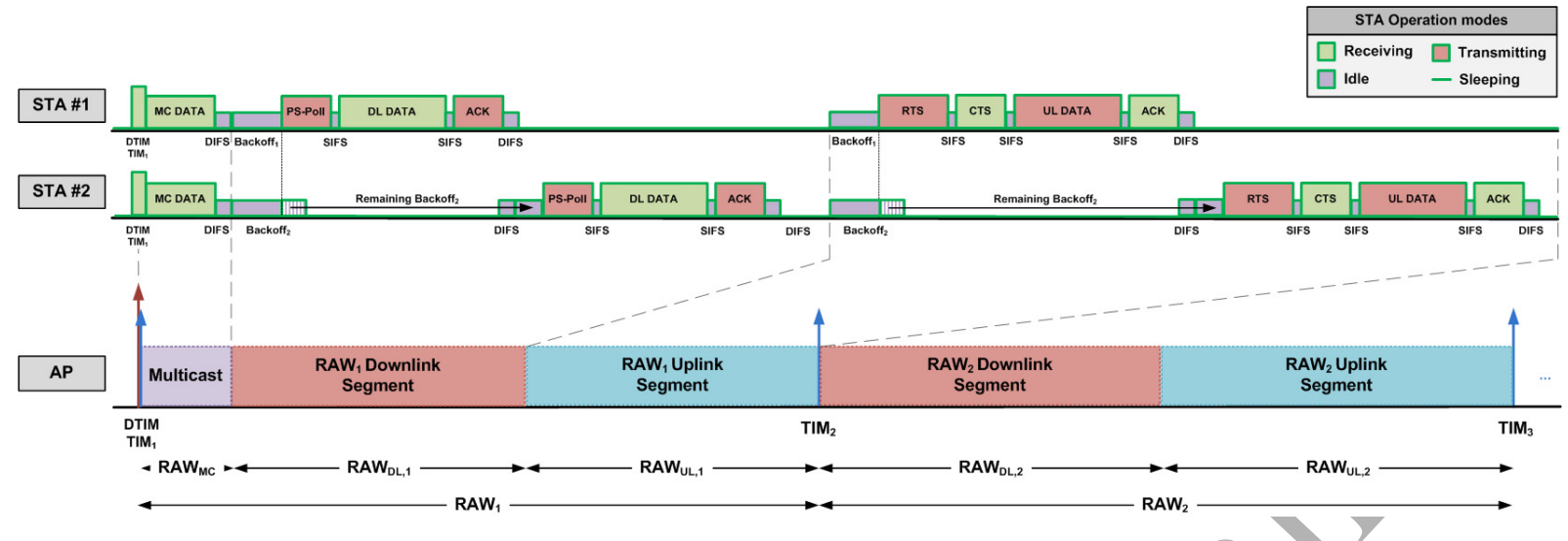

Figure 1: Beacon signalling and transmission procedures in IEEE 802.11ah WLANs using TUM and page'segmentation.

\subsection{Scenario}

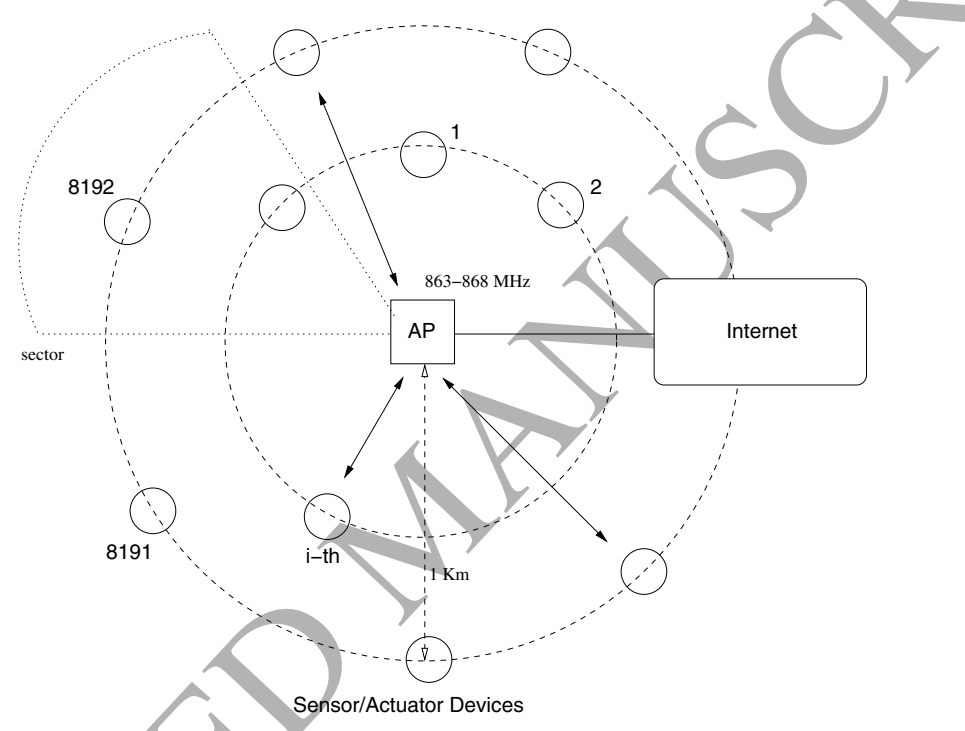

Figure 2: 1EEE 802.11ah scenario considered in this paper.

We consider an IEEE 802.11ah WLAN consisting of a number of stations $\left(N_{\mathrm{STA}}\right)$ randomly distributed over a given area, and a single AP placed at its center (see Figure 2). By applying STA sectorization, with as many sectors as TIM groups, it is guaranteed that any node is able to detect transmissions from other nodes in its own TIM group and, therefore, collisions with hidden nodes are not considered ${ }^{2}$.

\footnotetext{
${ }^{2} \operatorname{In}[14]$ and [15], the authors discuss the hidden node problem in IEEE 802.11ah networks, and they provide possible solutions to avoid this problem, e.g., the sectorization of STAs through TIM groups or the use of information from an AP to spread out UL transmissions over a period of time, thus eliminating the impact of hidden nodes.
} 


\subsubsection{Channel model}

STAs and the AP communicate at $R(d)$ rate, which depends on the distance between both devices and the environment's path loss. The approach presented in [16] is used to compute this value for both indoor and outdoor scenarios ${ }^{3}$ :

$$
R(d)=P_{\mathrm{TX}}+G_{\mathrm{TX}}-\mathrm{PL}(d)-\mathrm{FM}(d)+G_{\mathrm{RX}}-\left(\frac{E_{b}}{N_{0}}\right)_{d B}-N_{0}
$$

where $P_{T X}$ is the transmission power, $G_{T X}$ and $G_{R X}$ are the antenna gains at the transmitter and receiver, respectively, $\mathrm{PL}(d)$ is the path loss, $\mathrm{FM}(d)$ is the fade margin, $E_{b}$ is the energy required perinformation bit, $N_{0}$ is the thermal noise power density, and $\left(\frac{E_{b}}{N_{0}}\right)_{d B}$ value depends on the modulation and coding rate used. The different existing modulations and coding rates, as well as the rest of parameters used in this paper to compute the path loss, are shown in Table 1.

Transmitted packets can suffer from transmission errors with probability $p_{e}$. This value is assumed to be constant regardless the distance between the STA and the AP. This assumption is justified by the use of multiple transmission rates. We consider that the modulation and coding rate are adapted to compensate for the change in signal-to-noise ratio with the goal of keeping the actual packet error rate (PER) below the reference $p_{e}$ value $^{4}$. Moreover, it is only applied to DATA packets in UL. Other packets are considered error-free.

\subsubsection{Traffic model}

The probability of having a $\psi$ packet available for transmission $\left(p_{\psi}\right)$ within a DTIM period $(T)$ depends on the expected inter-packet time $\left(\mathrm{E}\left[T_{\mathrm{pck}_{\psi}}\right]\right)$. Since-we focus on low traffic load scenarios, we assume in all cases that $T \ll T_{\text {pck }_{\psi}}$, allowing us to simply compute $p_{\psi}$ as follows:

$$
p_{\psi}=\min \left(1, \frac{T}{\mathrm{E}\left[T_{\mathrm{pck}_{\psi}}\right]}\right)
$$

The number of STAs in an $\mathrm{RAW}_{\psi}$ segment with pending traffic (i.e., active stations competing for the channel access) is calculated as:

$$
A_{\psi}=p_{\psi} \cdot \frac{N_{\mathrm{STA}}}{N_{\mathrm{TIM}}}
$$

To keep the presented energy consumption model as simple as possible, we assume that packets unable to be transmitted in the same DTIM in which they were generated are discarded. As we will discuss in next

\footnotetext{
${ }^{3}$ For further details, we refer the reader to [16], where the calculation of $R(d)$ is explained in detail.

${ }^{4}$ Note that when using the channel model proposed in [16], the transmission rate used by a station, $R(d)$, is selected to guarantee that the actual experienced PER by a station is equal or lower than the reference value $p_{e}$. Therefore, in this paper we are considering a conservative approach in terms of PER.
} 
Table 1: Parameters of the channel propagation and IEEE 802.11ah PHY models.

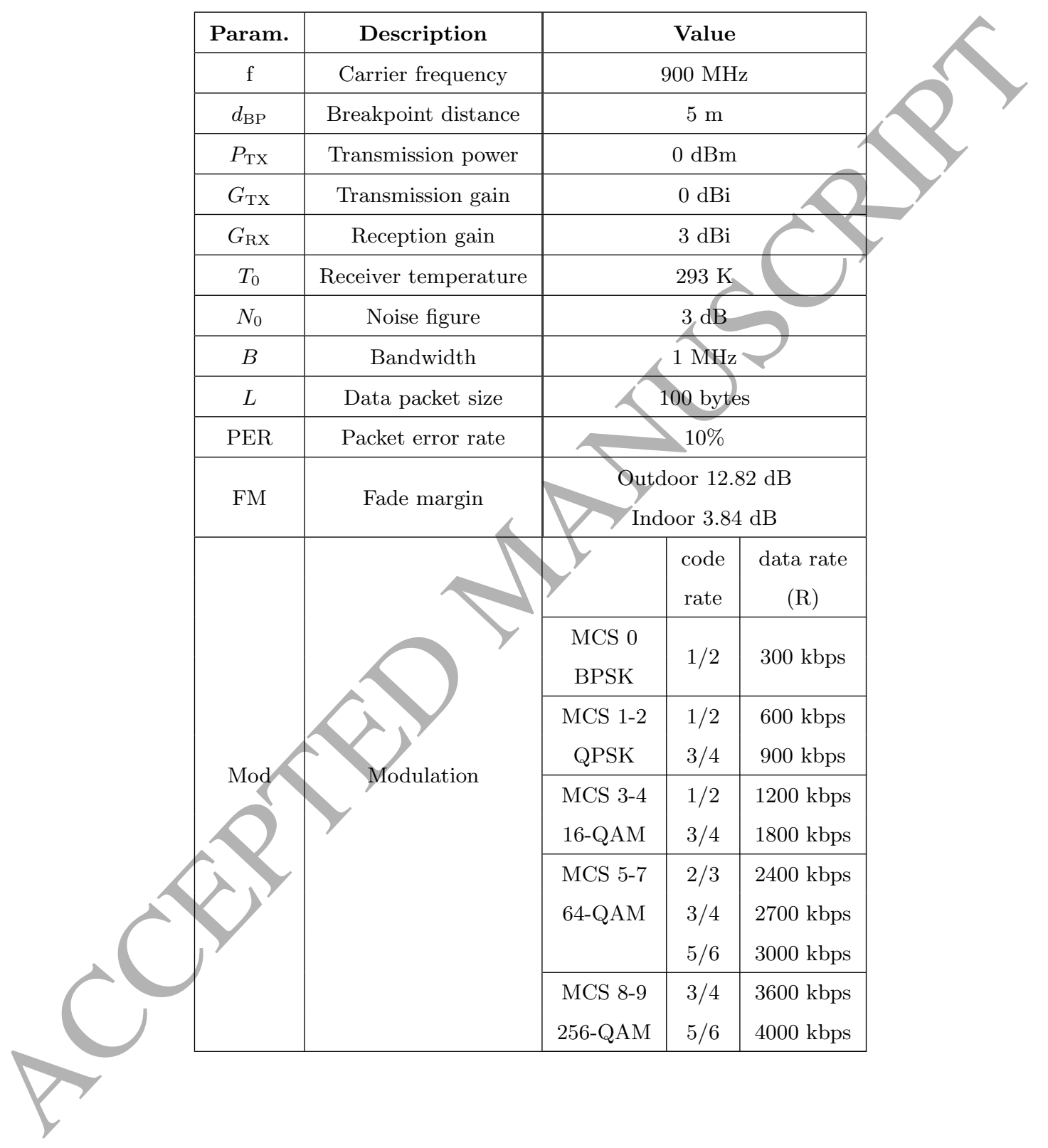


sections, not considering the queueing dynamics in the AP and STAs simplifies the complexity of the model without compromising its accuracy and applicability in typical operation conditions.

\section{Energy Consumption Model}

The energy consumption model proposed in this paper takes as a starting point the work of [10], and reformulates it by including the IEEE 802.11ah TIM and page segmentation elements that characterize the channel access.

As shown in Figure 1, within a DTIM period, an STA can perform the following actions:

- Listen to a DTIM beacon

- Listen to a TIM beacon

- Receive a multicast (MC) packet

- Receive a downlink (DL) packet

- Transmit an uplink (UL) packet

To perform these actions, an IEEE 802.11ah transceiver uses different operation modes $(\phi)$ for certain time periods: receiving $\left(t_{\mathrm{RX}}\right)$, transmitting $\left(t_{\mathrm{TX}}\right)$, idle $\left(t_{\mathrm{ID}}\right)$, and sleeping $\left(t_{\mathrm{SL}}\right)$.

Hence, the energy consumed $(E)$ by an IEEE 802.11ah STA (without considering data processing or sensor operation) is obtained by multiplying the time a transceiver is expected to be in each of its operation modes $\left(t_{\phi}\right)$, by their corresponding power consumption $\left(P_{\phi}\right)$ :

$$
E=t_{\mathrm{RX}} \cdot P_{\mathrm{RX}}+t_{\mathrm{TX}} \cdot P_{\mathrm{TX}}+t_{\mathrm{ID}} \cdot P_{\mathrm{ID}}+t_{\mathrm{SL}} \cdot P_{\mathrm{SL}}
$$

In the following lines, and after defining the main model parameters, we will calculate the energy consumed by an STA during a DTIM period according to the fraction of time it remains in each operation mode.

\subsection{Model definitions and assumptions}

We have only considered TIM stations [11], whose data transmissions must be performed within a RAW window with three differentiated segments (multicast, downlink and uplink), following a continuous delivery model [17]; i.e., nodes continuously transmit sensing data to the AP at a predefined rate. Other kind of traffic models, such as, event-driven [18], where data is generated after the activation of an alarm at the node, or query-driven, where the AP demands some actions to nodes, are not considered in our study. 
Table 2: Main IEEE 802.11ah energy consumption model parameters.

\begin{tabular}{|c|c|}
\hline Parameter & Description \\
\hline$T$ & DTIM beacon interval \\
\hline$N_{\mathrm{STA}}$ & Total number of network STAs \\
\hline$N_{\text {TIM }}$ & Number of TIM groups \\
\hline$\psi$ & Traffic type (DL, UL, or MC) \\
\hline$T_{\mathrm{pck}_{\psi}}$ & $\psi$ Packet generation time \\
\hline$A_{\psi}$ & Active STAs in an $\mathrm{RAW}_{\psi}$ segment \\
\hline$B_{\psi}$ & Active STAs in the beginning of each state (see Figure 3 ) \\
\hline$C_{\psi}$ & Number of STAs in an $\mathrm{RAW}_{\psi}$ segment that have previously accessed to the channel \\
\hline$r$ & Data rate \\
\hline$T_{\mathrm{DTIM}}$ & Duration of DTIM beacon (as defined in [11]) \\
\hline$T_{\text {TIM }}$ & Duration of TIM beacon (as defined in [11]) \\
\hline$T_{\mathrm{RAW}_{\psi}}$ & Duration of $\mathrm{RAW}_{\psi}$ segment (as defined in [11]) \\
\hline$p_{\psi}$ & Probability of an STA having a pending $\psi$ packet \\
\hline$p_{\text {DL }_{\text {TIM }}}$ & Probability of a TIM group having pending DL packets \\
\hline$p_{c_{\psi}}$ & Collision probability (only for PS-POLL or RTS frames) \\
\hline$p_{e_{\psi}}$ & Error probability (only for DATA frames) \\
\hline$p_{w_{\psi}}$ & Probability of an STA not crossing its corresponding $\mathrm{RAW}_{\psi}$ segment boundary \\
\hline$p_{f_{\psi}}$ & Percentage of free space in the own $\mathrm{RAW}_{\psi}$ after having been occupied by $C_{\psi}$ STAs \\
\hline$p_{s i, j}^{(\psi)}$ & Probability of an STA having a successful transmission after $i$ collisions and $j$ errors \\
\hline$p_{n s_{i, j}}^{(\psi)}$ & Probability of an STA not having a successful transmission after $i$ collisions and $j$ errors \\
\hline$\phi$ & Transceiver's operation mode (RX, TX, ID, or SL) \\
\hline$T_{i, j}^{\phi(\psi)}$ & $\begin{array}{l}\text { Time consumed in the } \phi \text { operation mode for a } \psi \text { transmission procedure } \\
\text { after } i \text { collisions and } j \text { errors (without reaching the } \mathrm{RAW}_{\psi} \text { segment boundary) }\end{array}$ \\
\hline$\hat{T}^{\phi(\psi)}$ & $\begin{array}{l}\text { Time consumed in the } \phi \text { operation mode for a } \psi \text { transmission procedure } \\
\text { (reaching the } \mathrm{RAW}_{\psi} \text { segment boundary) }\end{array}$ \\
\hline$\alpha, \beta, \gamma, \delta$ & Number of $T_{\text {DATA }}$ and $T_{c t r l}$ sending attempts in the $T_{i, j}^{\phi(\psi)}$ and $\hat{T}_{i, j}^{\phi(\psi)}$ equations \\
\hline$m_{\mathrm{col}}$ & $\begin{array}{l}\text { Retransmission limit for a PS_ POLL (DL transmission) } \\
\text { or an RTS frame (UL transmission) due to collisions }\end{array}$ \\
\hline$m_{\text {err }}$ & Retransmission limit for a DATA packet due to errors \\
\hline$t_{\text {slot }}$ & Duration of an IEEE 802.11ah time slot \\
\hline $\mathrm{CW}_{\min }$ & Minimum value of contention window \\
\hline $\mathrm{CW}_{\max }$ & Maximum value of contention window \\
\hline$T_{\text {DATA }}$ & Duration of a DATA frame \\
\hline$T_{c t r l}$ & Duration of a control frame (PS_POLL, ACK, RTS, or CTS) \\
\hline$T_{D I F}$ & Duration of a DIFS interval \\
\hline$T_{S I F S}$ & Duration of a SIFS interval \\
\hline$T_{\psi}$ & Duration of a $\psi$ successful transmission \\
\hline$T_{c_{\psi}}$ & Duration of a collision \\
\hline$T_{e_{\psi}}>$ & Duration of an error \\
\hline $\bar{T}_{o \psi}$ & Mean occupation time of an STA in its $\mathrm{RAW}_{\psi}$ segment \\
\hline
\end{tabular}


Table 3: Value of the variables involved in the state diagram according to the collision probability experienced in the contention round

$$
\begin{aligned}
& \begin{array}{|l|l|c|c|c|l|}
\hline & \begin{array}{l}
\text { Initial } \\
\text { state }
\end{array} & B_{\psi(i)} & \text { Transition } & \text { Next state } & C_{\psi(i)} \\
\hline \multirow{2}{*}{\text { Round } \# i} & S_{i} & A_{\psi} \cdot\left(\frac{p_{c_{\psi}}}{2}\right)^{i-1} & 1-p_{c_{\psi}} & S_{i}^{\prime} & \frac{B_{\psi(i)}}{2} \cdot\left(1-p_{c_{\psi}}\right) \\
\cline { 3 - 5 } & & p_{c_{\psi}} & S_{i+1} & \begin{array}{l}
B_{\psi(i)} \cdot\left(1-p_{c_{\psi}}\right)+ \\
B_{c_{\psi}}
\end{array} \\
\hline
\end{array} \\
& C_{\psi}=\frac{A_{\psi}}{2} \cdot\left(1-p_{c_{\psi}}\right)^{2}+\sum_{i=1}^{m_{\text {col }}-1} A_{\psi} \cdot\left(1-p_{c_{\psi}}\right) \cdot p_{c_{\psi}}^{i} \cdot\left(\left(\frac{p_{c_{\psi}}}{2}\right)^{i} \cdot \frac{1-p_{c_{\psi}}}{2}+\sum_{j=0}^{i-1}\left(\frac{p_{c_{\psi}}}{2}\right)^{j} \cdot\left(1-\frac{p_{c_{\psi}}}{2}\right)\right)+ \\
& +p_{c_{\psi}}^{m_{c o l}} \cdot\left(\sum_{i=0}^{m_{c o l}-1} A_{\psi} \cdot\left(\frac{p_{c_{\psi}}}{2}\right)^{i} \cdot\left(1-p_{c_{\psi}}\right)+A_{\psi} \cdot\left(\frac{p_{c_{\psi}}}{2}\right)^{i+1}\right)= \\
& =\frac{2^{-m_{c o l}} \cdot A_{\psi} \cdot\left[\left(\left(p_{c_{\psi}}^{2}-2\right) \cdot p_{c_{\psi}}^{m_{c o l}}-p_{c_{\psi}}^{2}+1\right) \cdot p_{c_{\psi}}^{m_{c o l}}+2^{m_{c o l}}\right]}{2-p_{c_{\psi}}^{2}}
\end{aligned}
$$

Any IEEE 802.11ah transmission procedure implies different time periods in the receiving, transmitting, and idle operation modes. Our model defines the $T_{i, j}^{\phi(\psi)}$ parameter, which computes these periods, being $i$ and $j$, respectively, the number of collisions and errors to perform that procedure. Similarly, $\hat{T}_{i, j}^{\phi(\psi)}$ computes those same periods when the transmission procedure reaches the $\mathrm{RAW}_{\psi}$ segment boundary without being successful. In both cases, $i$ and $j$ range from 0 to their maximum value $\left(m_{c o l}\right.$ and $m_{\text {err }}$, respectively) before the packet is dropped.

The channel contention rests on the DCF scheme. Collisions can occur when two or more STAs send at the same time a PS-POLL in a DL transmission procedure or an RTS frame in a UL transmission procedure, whereas errors are only confined to DATA frames. Collisions and errors produce a reset in the DL/UL transmission procedure and double the backoff window. The collision probability $p_{c_{\psi}}$ within an $\mathrm{RAW}_{\psi}$ segment containing $\frac{N_{S T A}}{N_{T I M}}$ STAs with $p_{\psi}$ traffic probability is given by:

$$
p_{c_{\psi}} \approx 1-\left(1-p_{\psi} \cdot \frac{1}{\mathrm{CW}_{\min }}\right)^{\frac{N_{\mathrm{STA}}}{N_{\mathrm{TIM}}}-1}
$$

The time an STA has to wait until accessing to the channel strongly depends on the number of STAs in the current $\mathrm{RAW}_{\psi}$ segment that, on average, have previously accessed to the channel $\left(C_{\psi}\right)$. To calculate this value, $\mathrm{RAW}_{\psi}$ segments are simplified by splitting them into rounds (i.e., time between two transmission attempts from the target STA), within which the considered STA competes for the channel. This estimation is modelled through the state diagram from Figure 3, where transitions between different states depend on $p_{c_{\psi}}$. Considering that there are $B_{\psi(i)}$ active STAs at the beginning of round $i, C_{\psi(i)}$ value is obtained from averaging the two possible options for STAs accessing the channel before the target STA (see Table 3): $i$ ) with $p_{c_{\psi}}$ probability, all STAs without collisions $\left(B_{\psi(i)} \cdot\left(1-p_{c}\right)\right)$ and half of those which have suffered them, 


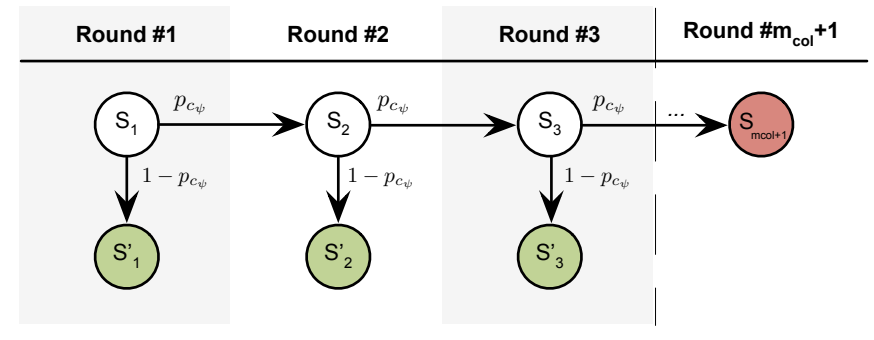

Figure 3: State diagram used to model the access to the channel of the target STA and its previous contenders $C_{\psi} \cdot S$ and $S^{\prime}$ states symbolize, respectively, the beginning and the success of the channel access in each contention round. $S_{m} m_{c o l}+1$ represents the STAs inability of acccessing to the channel in its corresponding $\mathrm{RAW}_{\psi}$

$\left(\frac{B_{\psi(i)}}{2} \cdot p_{c}\right)$, and $\left.i i\right)$ with $\left(1-p_{c}\right)$ probability, half of those $\operatorname{STAs}^{5}$ without collisions $\left(\frac{B_{\psi(i)}}{2} \cdot\left(1-p_{c}\right)\right)$. In our case, assuming that the target STA has a retransmission limit due to errors of $m_{\text {err }}=1$ and due to collisions of $m_{c o l}=7$, the process before described will be repeated until one of the limits is reached, i.e.:

- The considered STA is able to access to the channel (green states from Figure 3).

- The considered STA reaches the maximum retransmission limit $m_{c o l}$ (red state from Figure 3 ).

Therefore, by using the state diagram from Figure 3, it is possible to compute the probability of all possible combinations of events. Then, averaging them, $C_{\psi}$ is obtained (4).

The average channel occupation time $\left(\bar{T}_{o_{\psi}}\right)$ of these $C_{\psi}$ STAs is defined as:

$$
\begin{aligned}
\bar{T}_{o_{\psi}} & =\left(1-p_{c_{\psi}}\right) \cdot\left(1-p_{e_{\psi}}\right) \cdot T_{\psi} \\
& +p_{c_{\psi}} \cdot T_{c_{\psi}}+\left(1-p_{c_{\psi}}\right) \cdot p_{e_{\psi}} \cdot T_{e_{\psi}}
\end{aligned}
$$

with the following time values of a $\psi$ successful transmission $\left(T_{\psi}\right)$, a collision $\left(T_{c_{\psi}}\right)$, and an error $\left(T_{c_{\psi}}\right)$ :

$$
\begin{aligned}
T_{\mathrm{DL}}= & T_{\mathrm{PS} \_\mathrm{POLL}}+T_{\mathrm{SIFS}}+T_{\mathrm{DATA}}+T_{\mathrm{SIFS}}+T_{\mathrm{ACK}}+T_{\mathrm{DIFS}} \\
T_{\mathrm{UL}} & =T_{\mathrm{RTS}}+T_{\mathrm{SIFS}}+T_{\mathrm{CTS}}+T_{\mathrm{SIFS}}+T_{\mathrm{DATA}}+ \\
T_{\mathrm{DL}_{\mathrm{UL}}} & =T_{\mathrm{PS} \_\mathrm{POLL}}+T_{\mathrm{DIFS}} \\
T_{e_{\mathrm{DL}}} & =T_{\mathrm{RTS}}+T_{\mathrm{DIFS}} \\
T_{e_{\mathrm{UL}}} & =T_{\mathrm{RTS}}+T_{\mathrm{SIFS}}+T_{\mathrm{CTS}}+T_{\mathrm{SIFS}}+T_{\mathrm{DATA}}+T_{\mathrm{DIFS}}
\end{aligned}
$$

\footnotetext{
${ }^{5}$ This assumption is justified because in average, half of the stations will select a lower backoff value than the reference STA, and the other half a backoff value equal or higher.
} 
Due to the finite length of $\mathrm{RAW}_{\psi}$ segments, it is possible that an STA reaches the segment boundary without having successfully performed its own transmission procedure. The probability $p_{w_{\psi}}$ of having enough free space for a successful transmission after the channel being occupied by $C_{\psi}$ STAs is:

$$
p_{w_{\psi}}=1-\frac{C_{\psi} \cdot \bar{T}_{o_{\psi}}}{T_{\mathrm{RAW}_{\psi}}-T_{\psi}}
$$

Similarly, $p_{f_{\psi}}$ is the percentage of free space in the own $\mathrm{RAW}_{\psi}$ after having been occupied by $C_{\psi}$ STAs:

$$
p_{f_{\psi}}=1-\frac{C_{\psi} \cdot \bar{T}_{o_{\psi}}}{T_{\mathrm{RAW}_{\psi}}}
$$

The successful transmission probability of an STA is symbolized as $p_{s_{i, j}}^{(\psi)}$, where $i$ and $j$ are the number of previous collisions and errors, respectively.

$$
p_{s_{i, j}}^{(\psi)}=\left(\begin{array}{c}
i+j \\
i
\end{array}\right) \cdot p_{c_{\psi}}^{i} \cdot p_{e_{\psi}}^{j} \cdot\left(1-p_{c_{\psi}}\right)^{j+1} \cdot\left(1-p_{e_{\psi}}\right)
$$

Similarly, the probability of an STA not having a successful transmission after $i$ collisions and $j$ errors without crossing its corresponding $\mathrm{RAW}_{\psi}$ segment boundary is:

$$
p_{n s_{i, j}}^{(\psi)}=\left(\begin{array}{c}
i+\hat{j} \\
i
\end{array}\right) \cdot p_{c_{\psi}}^{i} \cdot p_{e_{\psi}}^{j} \cdot\left(1-p_{c_{\psi}}\right)^{j}
$$

From now on, Table 2 may be used as a reference, since it lists the main parameters considered in the model and their description.

\subsection{Consumption in the receiving state}

The time an STA is in the receiving state is given by (12), and includes the following situations:

(a) DTIM beacon transmission. Every DTIM beacon must be listened by all TIM STAs, since they contain all necessary information to send/receive data to/from the AP.

(b) TIM beacon/transmission. An STA listens to its corresponding TIM beacon with probability $p_{\text {DL }_{\text {TIM }}} \cup$ $p_{\mathrm{UL}}=p_{\mathrm{DL}_{\mathrm{TIM}}}+p_{\mathrm{UL}}-p_{\mathrm{DL}}{ }_{\mathrm{TIM}} \cdot p_{\mathrm{UL}}$, where $p_{\mathrm{DL}}=1-\left(1-p_{\mathrm{DL}}\right)^{\frac{N_{\mathrm{STA}}}{N_{\mathrm{TIM}}}}$ is the probability that the last DTIM beacon has informed of DL traffic for the TIM group to which the STA belongs.

(c) Multicast transmission. As observed in the receiving procedure in Figure 1, there is an RAW $\mathrm{MC}_{\mathrm{C}}$ segment placed immediately after every DTIM beacon. If previously signalled by the DTIM beacon, STAs remain in the receiving state to receive the corresponding MC packet. 
(d) Downlink data packet transmission. To receive a data packet, an STA remains in the receiving state for a certain time period called $T_{i, j}^{\mathrm{RX}(\mathrm{DL})}$, computed as a combination of three factors: the probability of successfully listening to the corresponding data packet $\left(p_{s_{i, j}}^{(D L)}\right)$, and the probabilities of the packet being

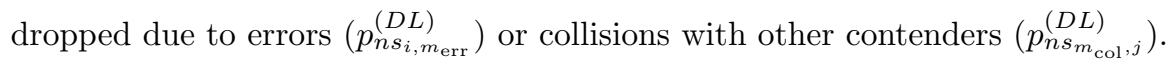

(e) Uplink data packet transmission. To receive the CTS and the ACK corresponding to a successful UL transmission, an STA remains in the receiving state for a certain time period called $T_{i, j}^{\mathrm{RX}(\mathrm{UL})}$. Similarly as in the previous case, this period also depends on the ability of accessing the channel, which can be affected by errors and collisions.

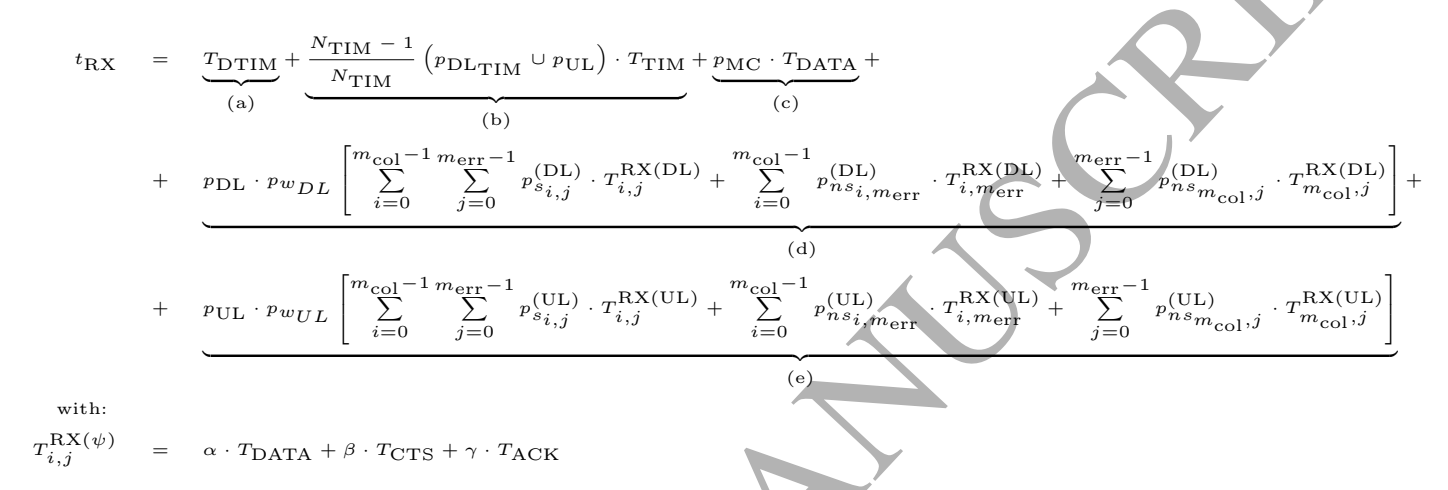

\subsection{Consumption in the transmitting state}

Table $4: \alpha, \beta$, and $\gamma$ values for (13)

\begin{tabular}{|c|c|c|c|c|c|c|}
\hline & \multicolumn{2}{|c|}{ DL transmission procedure } & \multicolumn{3}{c|}{ UL transmission procedure } \\
\hline & $T_{i, j}^{\mathrm{RX}(\mathrm{DL})}$ & $T_{i, m_{\mathrm{err}}}^{\mathrm{RX}(\mathrm{DL})}$ & $T_{m_{c \mathrm{ol}}, j}^{\mathrm{RX})}$ & $T_{i, j}^{\mathrm{RX}(\mathrm{UL})}$ & $T_{i, m_{\mathrm{err}}}^{\mathrm{RX}(\mathrm{UL})}$ & $T_{m_{\text {col }}, j}^{\mathrm{RX}(\mathrm{LL})}$ \\
\hline$\alpha$ & $j+1$ & $m_{\mathrm{err}}$ & $j$ & 0 & 0 & 0 \\
\hline$\beta$ & 0 & 0 & 0 & $j+1$ & $m_{\mathrm{err}}$ & $j$ \\
\hline$\gamma$ & 0 & 0 & 0 & 1 & 0 & 0 \\
\hline
\end{tabular}

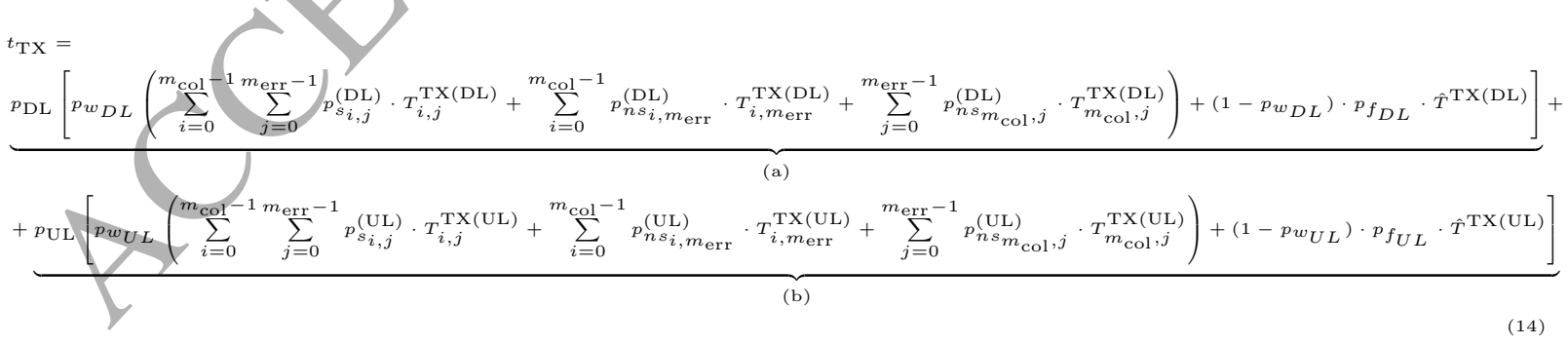

with:

$T_{i, j}^{\mathrm{TX}(\psi)}=\alpha \cdot T_{\mathrm{PS}}$ POLL $+\beta \cdot T_{\mathrm{ACK}}+\gamma \cdot T_{\mathrm{RTS}}+\delta \cdot T_{\mathrm{DATA}}$ 
Table 5: $\alpha, \beta, \gamma$, and $\delta$ values for (15)

\begin{tabular}{|c|c|c|c|c|c|c|c|c|}
\hline & \multicolumn{4}{|c|}{ DL transmission procedure } & \multicolumn{4}{c|}{ UL transmission procedure } \\
\hline & $T_{i, j}^{\mathrm{TX}(\mathrm{DL})}$ & $T_{i, m_{\mathrm{err}}}^{\mathrm{TX}(\mathrm{DL})}$ & $T_{m_{\mathrm{col}}, j}^{\mathrm{TX}(\mathrm{DL})}$ & $\hat{T}^{\mathrm{TX}(\mathrm{DL})}$ & $T_{i, j}^{\mathrm{TX}(\mathrm{UL})}$ & $T_{i, m_{\mathrm{err}}}^{\mathrm{TX}(\mathrm{UL})}$ & $T_{m_{\text {col }}, j}^{\mathrm{TX}(\mathrm{UL})}$ & $\hat{T}^{\mathrm{TX}(\mathrm{UL})}$ \\
\hline$\alpha$ & $i+j+1$ & $i+m_{\mathrm{err}}$ & $m_{\mathrm{col}+j}$ & 1 & 0 & 0 & 0 & 0 \\
\hline$\beta$ & 1 & 0 & 0 & 0 & 0 & 0 & 0 & 0 \\
\hline$\gamma$ & 0 & 0 & 0 & 0 & $i+j+1$ & $i+m_{\mathrm{err}}$ & $m_{\mathrm{col}+j}$ & 1 \\
\hline$\delta$ & 0 & 0 & 0 & 0 & $j+1$ & $m_{\mathrm{err}}$ & $j$ & 0 \\
\hline
\end{tabular}

The time an STA is in the transmitting state is given by (14), and includes the following situations:

(a) Downlink reception. To complete a successful DL data reception, STAs also have to send a PS-POLL and an ACK frame. The length of the time period in the transmitting state $\left(T_{i, j}^{\mathrm{TX}(\mathrm{DL})}\right)$ varies as a function of the PS-POLL sending success, which depends in turn on transmission errors, collisions, and the RAW $\mathrm{DL}_{\mathrm{DL}}$ size.

(b) Uplink transmission. The time an STA remains in the transmitting state $\left(T_{i, j}^{\mathrm{TX}(\mathrm{UL})}\right)$ is not only due to the sending of DATA frames, but also of RTS frames. Although both transmissions can be affected by errors, only RTS frames can suffer collisions.

\subsection{Consumption in the idle state}

The time an STA is in the idle state is given by (16), and includes the following situations:

(a) Multicast reception. After receiving a multicast packet, all STAs go to sleep except those from the first TIM group to which the AP has pending data to send, staying in the idle state for the duration of the subsequent DIFS.

(b) Downlink reception. Time in the idle state $\left(T_{i, j}^{\mathrm{ID}(\mathrm{DL})}\right)$ is modelled as an addition of DIFS, SIFS, backoff, and waiting periods due to the data reception procedure of other TIM group contenders. It is assumed in the model that, on average, any STA transmits after the channel has been occupied by $C_{\psi}$ STAs,

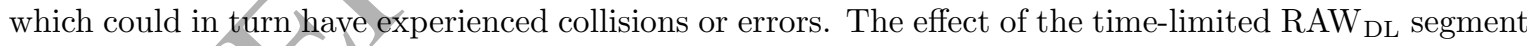
is also considered.

(c) Uplink transmission. Similarly, time in the idle state $\left(T_{i, j}^{\mathrm{ID}(\mathrm{UL})}\right)$ is computed as an addition of DIFS, SIFS, backoff, and waiting periods due to the data transmission procedure of the other $C_{\psi}$ STAs. 
Table 6: $\alpha, \beta$, and $\gamma$ values for (17)

\begin{tabular}{|c|c|c|c|c|c|c|}
\hline & \multicolumn{3}{|c|}{ DL transmission procedure } & \multicolumn{3}{c|}{ UL transmission procedure } \\
\hline & $T_{i, j}^{\mathrm{ID}(\mathrm{DL})}$ & $T_{i, m_{\mathrm{err}}}^{\mathrm{ID}(\mathrm{DL})}$ & $T_{m_{\mathrm{col}}, j}^{\mathrm{ID}(\mathrm{DL})}$ & $T_{i, j}^{\mathrm{ID}(\mathrm{UL})}$ & $T_{i, m_{\mathrm{err}}}^{\mathrm{ID}(\mathrm{UL})}$ & $T_{m_{\mathrm{col}}, j}^{\mathrm{ID}(\mathrm{UL})}$ \\
\hline$\alpha$ & $i+j+1$ & $i+m_{\mathrm{err}}$ & $m_{\mathrm{col}}+j$ & $i+j+1$ & $i+m_{\mathrm{err}}$ & $m_{\mathrm{col}}+j$ \\
\hline$\beta$ & $j+2$ & $m_{\mathrm{err}}$ & $j$ & $2 j+3$ & $2 m_{\mathrm{err}}$ & $2 j$ \\
\hline$\gamma$ & $i+j$ & $i+m_{\mathrm{err}}-1$ & $m_{\mathrm{col}}+j-1$ & $i+j$ & $i+m_{\mathrm{err}}-1$ & $m_{\mathrm{col}}+j-1$ \\
\hline
\end{tabular}

$$
\begin{aligned}
& t_{\mathrm{ID}} \approx \underbrace{p_{\mathrm{MC}} \cdot T_{\mathrm{DIFS}}}_{\text {(a) }}+ \\
& \underbrace{p_{\mathrm{DL}}\left[p_{w_{D L}}\left(\sum_{i=0}^{m_{\mathrm{col}}-1} \sum_{j=0}^{m_{\mathrm{err}}^{-1}} p_{s_{i, j}}^{(\mathrm{DL})} \cdot T_{i, j}^{\mathrm{ID}(\mathrm{DL})}+\sum_{i=0}^{m_{\mathrm{col}}^{-1}} p_{n s_{i, m} \mathrm{err}}^{(D L)} \cdot T_{i, m_{\mathrm{err}}}^{\mathrm{ID}(\mathrm{DL})}+\sum_{j=0}^{m_{\mathrm{err}}^{-1}} p_{n s_{m}(\mathrm{DL})}^{m_{\mathrm{col}}, j} \cdot T^{m_{\mathrm{col}}, j}\right)\right]+}_{(\mathrm{b})} \\
& \underbrace{+p_{\mathrm{DL}}\left[\left(1-p_{w_{D L}}\right) \cdot\left(T_{\mathrm{RAW}}-p_{f_{D L}} \cdot \hat{T}^{\mathrm{TX}(\mathrm{DL})}\right)\right]}_{\text {(b) }}+
\end{aligned}
$$

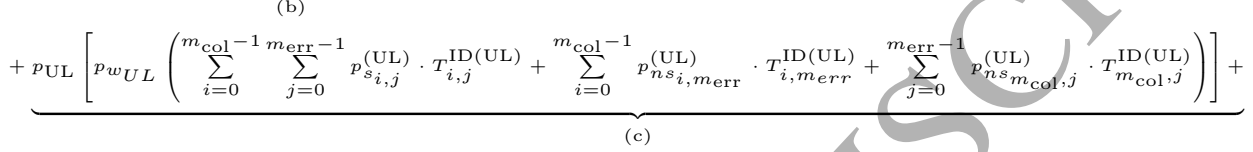

$$
\begin{aligned}
& \underbrace{+p_{\mathrm{UL}}\left[\left(1-p_{w_{U L}}\right) \cdot\left(T_{\mathrm{RAW}_{U L}}-p_{f_{U L}} \cdot \hat{T}^{\mathrm{TX}(\mathrm{UL})}\right)\right]}_{\text {(c) }} \\
& T_{i, j}^{\mathrm{ID}(\psi)}=\alpha \cdot T_{\mathrm{DIFS}}+\beta \cdot T_{\mathrm{SIFS}}+t_{\mathrm{slot}} \cdot\left(\sum_{k=0}^{\gamma} \frac{\min \left\{2^{k} \cdot\left(\mathrm{CW}_{\min }+1\right), \mathrm{CW}_{\max }+1\right\}}{2}\right) \\
& +C_{\psi} \cdot\left(\left(1-p_{c_{\psi}}\right) \cdot\left(1-p_{e_{\psi}}\right) \cdot T_{\psi}+p_{c_{\psi}} \cdot T_{c_{\psi}}+\left(1-p_{c_{\psi}}\right) \cdot p_{e_{\psi}} \cdot T_{e_{\psi}}\right)
\end{aligned}
$$

\subsection{Consumption in the sleeping state}

Finally, an STA remains asleep when not being in any other state:

$$
t_{\mathrm{SL}}=T-t_{\mathrm{RX}}-t_{\mathrm{TX}}-t_{\mathrm{ID}}
$$

\section{Model Evaluation}

This section provides a comparative analysis between the proposed energy consumption analytical model and the results obtained from simulating a fully connected IEEE 802.11ah WLAN in MATLAB, where the system model introduced in Section 2 is accurately implemented. For both, model and simulation, main MAC layer parameters are summarized in Table 7. The results presented contribute to understand the performance of the TIM and page segmentation mechanism included in the IEEE 802.11ah amendment.

In the simulator, collisions happen if two or more STAs from the same TIM group compute the same backoff value. After a collision, all those involved STAs select another random backoff value after doubling 
Table 7: Main parameters for IEEE 802.11ah MAC layer

\begin{tabular}{|c|l|c|l|}
\hline$t_{\text {simulation }}$ & $T=1.6 \mathrm{~s}$ & $m_{\text {err }}$ & 1 \\
\hline$N_{\text {TIM }}$ & 8 & $L_{\text {DATA }}$ & 100 bytes \\
\hline$T_{\text {SIFS }}$ & $160 \mu s$ & $L_{\text {PS_POLL }}$ & 14 bytes \\
\hline$T_{\text {DIFS }}$ & $264 \mu s$ & $L_{\text {ACK }}$ & 14 bytes \\
\hline$t_{\text {slot }}$ & $52 \mu s$ & $L_{\text {RTS }}$ & 20 bytes \\
\hline $\mathrm{CW}_{\min }$ & 16 & $L_{\mathrm{CTS}}$ & 14 bytes \\
\hline $\mathrm{CW}_{\max }$ & 1024 & $p_{e_{\mathrm{DL}}}$ & 0 \\
\hline$m_{\text {col }}$ & 7 & $p_{e_{\mathrm{UL}}}$ & 0.1 \\
\hline
\end{tabular}

the $\mathrm{CW}_{\min }$ value. Moreover, we have assumed, as in the model, that the uplink data packets suffer errors with a probability of $10 \%\left(p_{e_{U L}}=0.1\right)$.

The main difference of the model with respect to the simulator is that in the latter one STAs accumulate in a buffer all those packets not transmitted in their corresponding RAW $\psi$ segment, having the opportunity of sending them in subsequent ones. Comparing the simulation results with the ones obtained from the model also allow us to quantify the impact of such an assumption.

\subsection{Comparison of different traffic patterns}

First simulation results show a comparison of the mean current consumption per STA in an IEEE 802.11ah network according to the analytical model, the simulator, and the simulator without buffer. Three different traffic patterns $\left(p_{\mathrm{DL}}=p_{\mathrm{UL}}=\{25 \%, 15 \%, 5 \%\}\right)$ and two scenarios based on the models presented in subsection $2.2($ a $100 m \times 100 m$ indoor network and $1000 m \times 1000 m$ outdoor network $)$ have been considered.

Mean current consumption $(\bar{I})$ has been obtained as a ponderation of the different current consumptions,

$$
\bar{I}=\frac{t_{\mathrm{RX}} \cdot I_{\mathrm{RX}}+t_{\mathrm{TX}} \cdot I_{\mathrm{TX}}+t_{\mathrm{ID}} \cdot I_{\mathrm{ID}}+t_{\mathrm{SL}} \cdot I_{\mathrm{SL}}}{t_{\mathrm{RX}}+t_{\mathrm{TX}}+t_{\mathrm{ID}}+t_{\mathrm{SL}}}
$$

with the specific values used for this evaluation extracted from the CC1100 transceiver datasheet [19]: $I_{\mathrm{RX}}=15.5 \mathrm{~mA}, I_{\mathrm{TX}}=17.04 \mathrm{~mA}, I_{\mathrm{ID}}=1.6 \mathrm{~mA}$, and $I_{\mathrm{SL}}=0.9 \mu \mathrm{A}$. Figure 4 and Figure 5 show the results of the mean current consumption for the above considered cases.

One can observe that analytical results in both scenarios are very similar to those obtained by simulation, except when there exists a high number of packets in the network. This situation, caused by high traffic load and/or high number of STAs, leads to an increase of the collision probability and, consequently, to the impossibility of successfully transmitting all generated packets.

The presence of a buffer in the simulator allows STAs to retry the transmission of a packet in a next DTIM, thus filling this time period with more active STAs competing for the channel and dramatically 


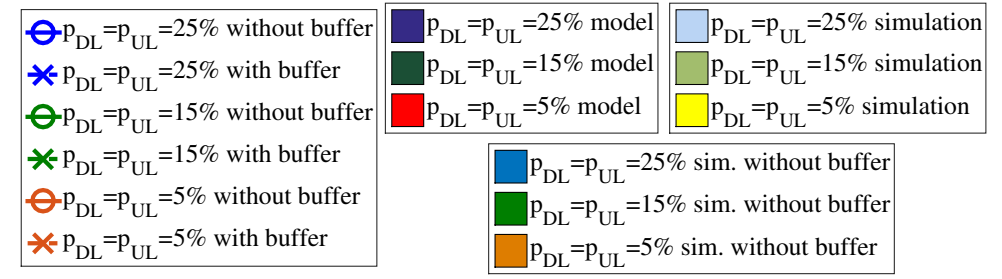

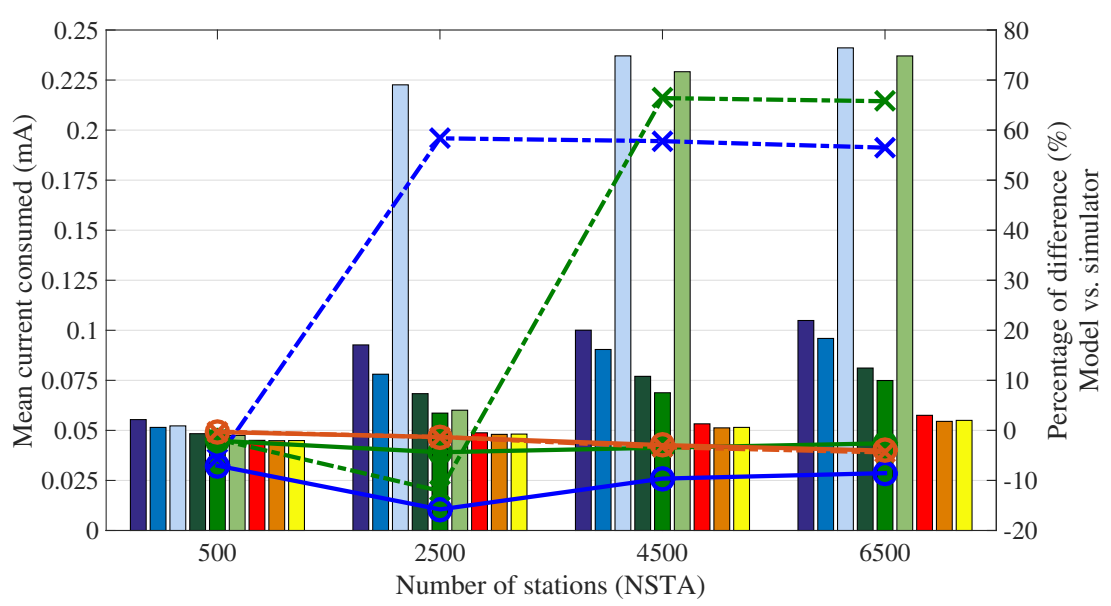

Figure 4: Mean current consumption per STA in an IEEE 802.11ah network located in an indoor scenario for different packet generation rates.

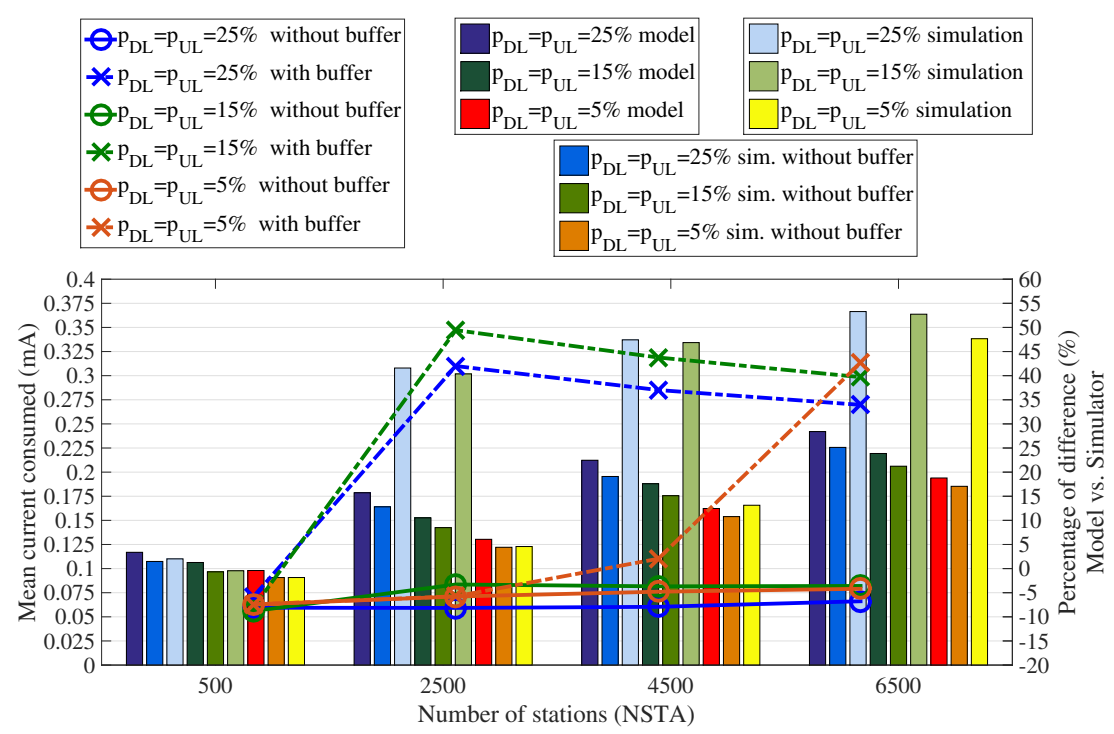

Figure 5: Mean current consumption per STA in an IEEE 802.11ah network located in an outdoor scenario for different packet generation rates. 

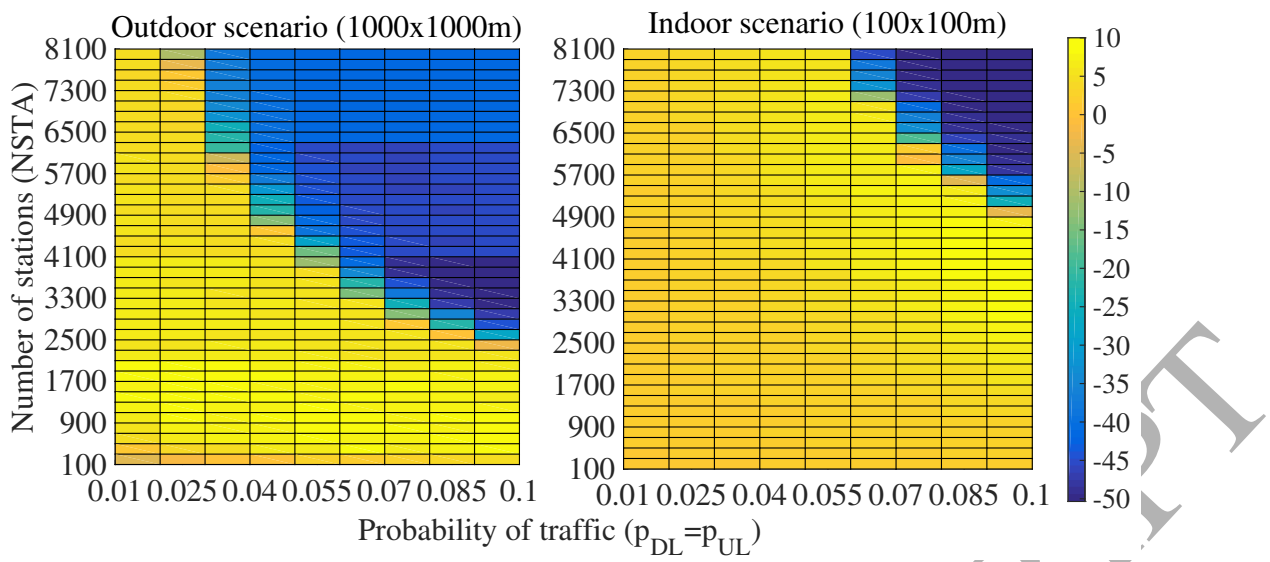

Figure 6: Percentage of difference mean current consumed by model vs. simulator for different number of stations and different generation rates (the range of "probability of traffic" corresponds to an expected generation time from $160 \mathrm{~s}$ to $16 \mathrm{~s}$, where $p_{\psi}$ is computed from $(2))$.

increasing their current consumption. As expected, these differences are not so acute when comparing the analytical model and the simulator without buffer.

The same comparison between the simulator and the model has been repeated for multiple combinations of traffic load and number of STAs, resulting in the maps of Figure 6. From these results, it is possible to determine the area in which our model is accurate; i.e., the validity area, defined as those combinations of parameters where the maximum deviation of current consumption is below $10 \%$ (note the validity area of the model in yellowish colors).

For the outdoor scenario, traffic loads above $10 \%$ place the validity area below 2500 STAs. However, in the indoor scenario this value almost doubles: 4900 STAs. This large difference is due to the propagation model assumed, as the lower the distance STA-AP, the higher the data rate that the STA will use. As STAs in the indoor scenario are closer to the AP, they can assume a higher data rate and, therefore, a higher number of transmissions can be allocated in every RAW segment.

\subsection{Results from four different application scenarios}

In order to validate the model accuracy in different representative IoT scenarios, we have considered the four use cases (agricultural monitoring, smart metering, industrial automation and animal monitoring) already presented in $[12]$ and summarized at Table 8.

Figure 7 shows that all four considered scenarios fall into the validity area, and the high accuracy between the model and the simulations in terms of mean current consumption. Among the different scenarios, the greatest deviation (nearly 5\%) appears in the agricultural one, because it contains the highest number of stations and, consequently, the highest number of packets to transmit. In that sense, the high collision probability boosts the chances of a packet not being transmitted in its corresponding $\mathrm{RAW}_{\psi}$ segment. 
Table 8: Main simulation parameters for four applications scenarios.

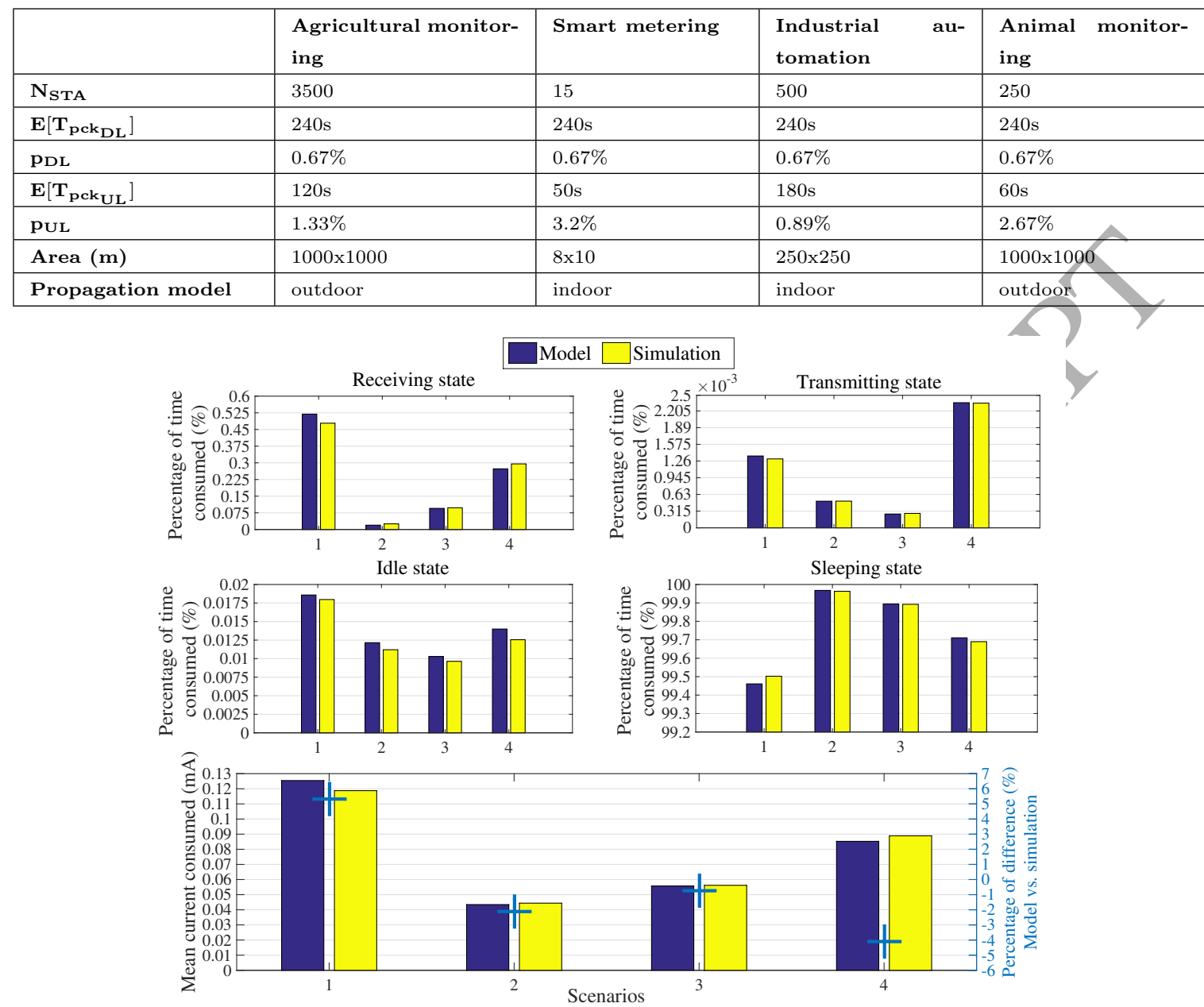

1: Agricultural monitoring 2: Smart metering 3: Industrial automation 4: Animal monitoring Figure 7: Percentage of consumption in each real scenario.

In addition to the problem of not considering a buffer in our model, one can observe in Figure 7 that the major differences between the model and the simulations are obtained in the receiving and idle states.

- Idle state: The idle state highly depends on the time spent while waiting for accessing to the channel. The proposed model overestimates the collision probability compared to simulations (see Figure 8), which results in a higher number of STAs that transmit before our target STA, and consequently, more time spent while waiting for accessing to the channel (idle state).

- Receiving state: Our model also presents differences at the receiving state in the agricultural scenario. In this case, the high number of STAs per TIM group affects the way we estimate the probability of listening to TIM beacons $\left(p_{\mathrm{DL}_{\mathrm{TIM}}}\right)$, resulting in greater energy consumption. However, this effect has no impact on the other scenarios, due to the lower density of active STAs per TIM group. 

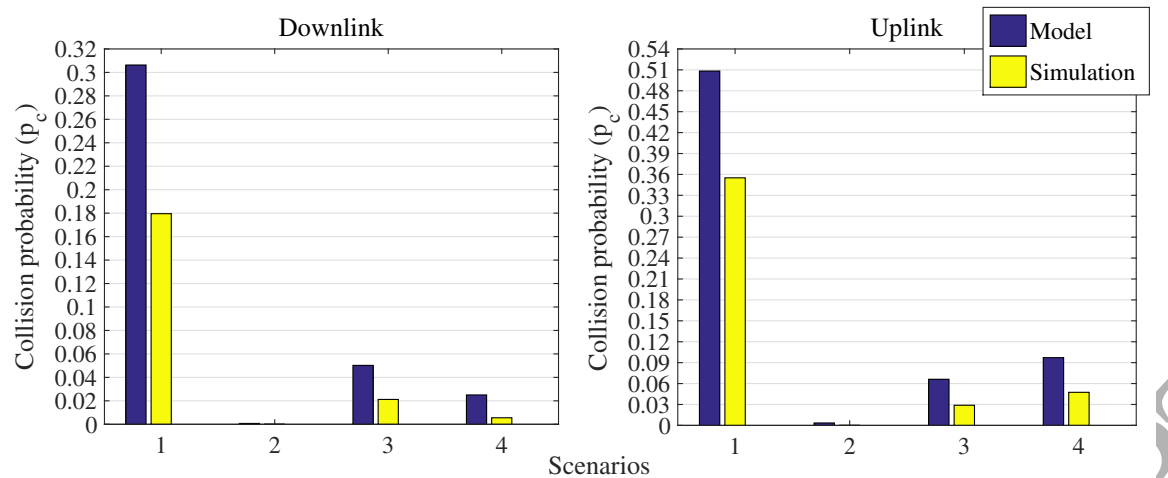

(1: Agricultural monitoring 2: Smart metering 3:Industrial automation 4: Animal monitoring)

Figure 8: Collision pobability $p_{c}$ in each real scenario.

In the other scenarios (mostly at the animal monitoring one, with the second largest uplink traffic load) the model gives us lower consumption values than simulations. As some of the packets may not be transmitted in their corresponding TIM period, the simulator will try to retransmit them in the following TIM periods; which is not considered by the model due to its lack of buffer (i.e., those packets are discarded). The fact of transmitting packets in subsequent segments requires to listen to more beacons, thus increasing the consumption at the receiving state.

- Transmitting state: The fact of not transmitting a packet in its corresponding TIM period, or estimating a higher collision probability, do not have a deep impact at the transmitting state. Therefore, it does not result in major differences between the model and simulations.

- Sleeping state: The sleeping state is obtained in (18) from the estimated values of the rest of the states and therefore reflects the estimation differences of the other states.

Lastly, with the proposed model, it is possible to predict the energy that a network will consume and even estimate its overall battery lifetime $\left(B_{\mathrm{LT}}\right)$ :

$$
B_{\mathrm{LT}}=\frac{C}{\bar{I}}
$$

where $C$ is the capacity of the battery in mAh.

In Figure 9 we compare the battery lifetime obtained from the simulator and the model. Both values are very similar (less than $1 \%$ of difference). The major difference obtained is roughly half a year when 2 AA batteries are used.

\section{Impact of system parameters}

Two parameters define the channel access in an IEEE 802.11ah network: the number of TIM groups in which stations are distributed $\left(N_{\text {TIM }}\right)$ and time between two consecutive DTIM beacons $(T)$. Their influence 

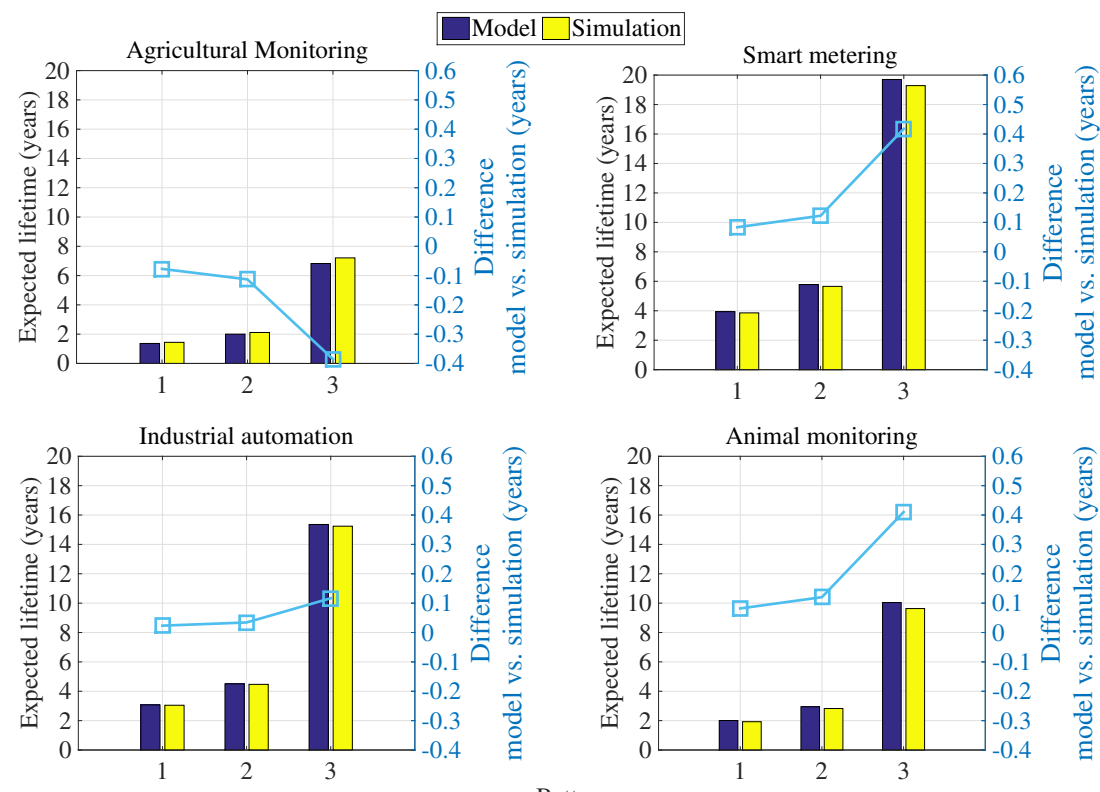

1: 2xAAA Batteries (1550 mAh) 2: Lithium Battery (2200 mAh) 3: 2xAA Batteries (7500 $\mathrm{mAh})$

Figure 9: Battery lifetime estimation for the four considered scenarios.

on the performance of an IEEE 802.11ah network for the four previously considered scenarios is studied in the following lines.

\subsection{Number of TIM groups $\left(N_{T I M}\right)$}

We can observe in Figure 10 that, in terms of consumed current, $N_{\text {TIM }}=8$ is the best configuration for several values of $T$. Although increasing the number of TIM groups reduces collisions, the current consumption is noticeably affected by the larger size of beacons.

\subsection{DTIM beacon interval (T)}

Results shown in Figure 11 reflect that the higher the value of $T$, the lower the current consumption. However, if $T$ is increased, the time between two transmission opportunities also does (i.e., an STA can only transmit in its own $\mathrm{RAW}_{\psi}$ segment), which is a limiting factor when there is a deadline for transmitting a newly generated packet. As it can be seen in Figure 11, for a fixed value of $N_{\text {TIM }}=8$, the higher the value of $\mathrm{T}$, the lower the current consumed, but the higher the delay when transmitting packets.

Unlike $N_{\text {TIM }}$, it is not possible to find an optimum value of $T$ and it is necessary to achieve a trade-off between the energy consumption and the delay related to a packet transmission. 

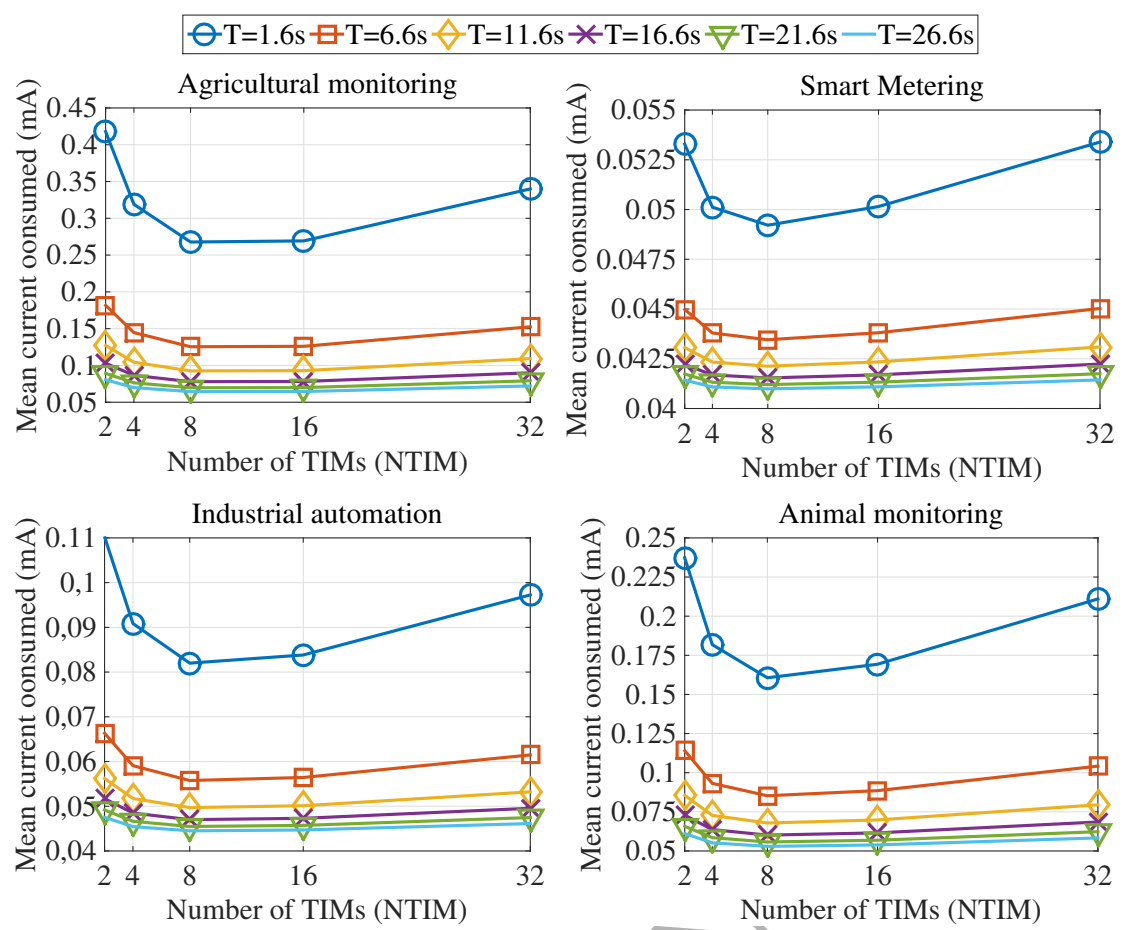

Figure 10: Current consumed vs. number of TIM groups $\left(N_{\mathrm{TIM}}\right)$.

\section{Conclusions}

It is a well-known fact that energy consumption represents one of the most striking challenges in the design and exploitation of LPWANs. The study and characterization of this behaviour according to different network conditions such as traffic load or number of stations, as well as other intrinsic network parameters, becomes therefore an essential step previous to further research in energy-saving mechanisms.

In this work, an analytical model to characterize the energy consumption of an IEEE 802.11ah station has been proposed. Its accuracy has been proved by comparing it with simulation results from four representative M2M scenarios. In all of them the model has been an excellent tool to estimate their battery lifetime.

For the network and system settings used in the study, $N_{\text {TIM }}=8$ was found to minimize the energy consumption for the wide range of scenarios considered. Unlike the $N_{\text {TIM }}$ parameter, $T$ should be selected depending on the seenario, because high values always reduce energy consumption but increase delay, resulting in a trade-off between these two metrics. With this study, our model proves its usefulness when planning an IEEE 802.11ah network, as it provides a reliable approximation of its energy consumption. Based on a set of closed-form equations, our model allows to quickly find the most suitable values for the different parameters in any desired scenario.

The current model may be extended in order to include some of the latest IEEE 802.11ah MAC features intended to support energy-efficient communications for sensors. Among them, [20] outlines the most notable 

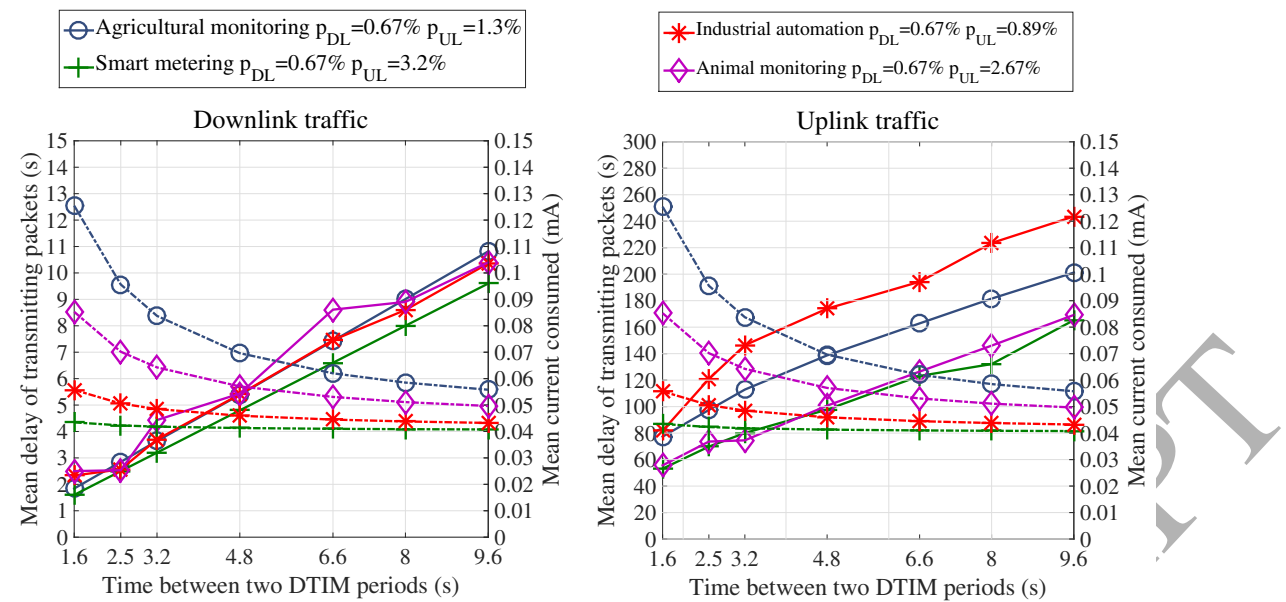

Figure 11: Comparison between mean delay and mean current consumed vs. time between two consecutive DTIM beacons (T seconds).

ones: Bidirectional TXOP allows for exchanging one or more UL and DL packets in a single transmission opportunity (TXOP) duration, NDP CMAC (Null Data Packet Carrying MAC) reduces overhead of control frames, and Short MAC Frame does the same with MAC headers. Lastly, this model opens the door to further research in the design of advanced power saving mechanisms which will help to enlarge the battery lifetime of sensor nodes while ensuring proper network operation.

While this paper focus on the IEEE 802.11ah link-layer mechanisms, future works should place more attention to charaterize the effects of different channel / PHY models on the performance of LPWANs, including the capture effect, as they may have also significant effects in the network performance, hence complementing the results presented in this paper.

\section{Acknowledgments}

This work was partially supported by the Spanish and Catalan governments through the projects TEC201679510-P and SGR-2014-1173 (both with FEDER funds), respectively. It has also been funded by the ENTOMATIC FP7-SME-2013 EC project (605073).

\section{References}

[1] Machina Research, The need for low cost, high reach, wide area connectivity for the Internet of Things, http://www.neul.com/neul/wp-content/uploads/2014/02/Neul_Machina_Research_White_Paper.pdf, accessed: 2015-11-20, 2014. 
[2] IEEE 802.11ah: Wireless LAN Medium Access Control (MAC) and Physical Layer (PHY) Specifications Amendment 2: Sub 1 GHz License Exempt Operation, IEEE Std 802.11ah-2016 (Amendment to IEEE Std 802.11-2016, as amended by IEEE Std 802.11ai-2016) (2017) 1-565.

[3] S. Aust, R. V. Prasad, I. G. Niemegeers, IEEE 802.11ah: Advantages in Standards and Further Challenges for Sub $1 \mathrm{GHz} \mathrm{Wi}-\mathrm{Fi}$, in: Proceedings of IEEE International Conference on Communications, ICC 2012, 6885-6889, 2012.

[4] B. Bellalta, L. Bononi, R. Bruno, A. Kassler, Next Generation IEEE 802.11 Wireless Local Area Networks: Current Status, Future Directions and Open Challenges, Computer Communications .

[5] E. Khorov, A. Lyakhov, A. Krotov, A. Guschin, A survey on IEEE 802.11 ah: An enabling networking technology for smart cities, Computer Communications 58 (2015) 53-69.

[6] C. G. et al, TIM and Page Segmentation, https://mentor.ieee.org/802.11/den/12/11-12-1084-04-00ahtim-and-page-segmentation.ppt, 2012.

[7] IEEE Standard for Information technology-Telecommunications and information exchange between systems Local and metropolitan area networks-Specific requirements Part 11: Wireless LAN Medium Access Control (MAC) and Physical Layer (PHY) Specifications, IEEE Std 802.11-2012 (Revision of IEEE Std 802.11-2007) (2012) 1-2793doi:10.1109/IEEESTD.2012.6178212.

[8] L. Zheng, L. Cai, J. Pan, M. Ni, Performance analysis of grouping strategy for dense IEEE 802.11 networks, in: Global Communications Conference (GLOBECOM), 2013 IEEE, 219-224, doi: 10.1109/GLOCOM.2013.6831074, 2013.

[9] R. P. Liu, G. Sutton, I. Collings, Power save with Offset Listen Interval for IEEE 802.11ah Smart Grid communications, in: Communications (ICC), 2013 IEEE International Conference on, ISSN 1550-3607, 4488-4492, doi:10.1109/ICC.2013.6655274, 2013.

[10] O. Raeesi, J. Pirskanen, A. Hazmi, T. Levanen, M. Valkama, Performance evaluation of IEEE 802.11ah and its restricted access window mechanism, in: Communications Workshops (ICC), 2014 IEEE International Conference on, 460-466, doi:10.1109/ICCW.2014.6881241, 2014.

[11] T. Adame, A. Bel, B. Bellalta, J. Barcel, J. Gonzalez, M. Oliver, Capacity Analysis of IEEE 802.11ah WLANs for M2M Communications., in: MACOM, vol. 8310 of Yecture Notes in Computer Science, Springer, ISBN 978-3-319-03870-4, 139-155, URL http://dblp.uni-trier.de/db/conf/macom/macom2013.html, 2013. 
[12] T. Adame, A. Bel, B. Bellalta, J. Barcelo, M. Oliver, IEEE 802.11AH: the WiFi approach for M2M communications, Wireless Communications, IEEE 21 (6) (2014) 144-152, ISSN 1536-1284, doi: 10.1109/MWC.2014.7000982.

[13] A. Bel, T. Adame, B. Bellalta, J. Barcelo, J. Gonzalez, M. Oliver, CAS-based Channel Access Protocol for IEEE 802.11ah WLANs, European Wireless 2014; 20th European Wireless Conference; Proceedings of, May 2014.

[14] M. P. et al, Uplink Channel Access, https:// mentor.iee.org/802.11/dcn/12/11-12-0606-01-00ah-uplinkchannel-access.pptx, 2012.

[15] G. G. et al, Sectorization for hidden node mitigation, https://mentor.ieee.org/802.11/dcn/12/11-120852-00-00ah-sectorization-for-hidden-node-mitigation.pptx, 2012.

[16] A. Hazmi, J. Rinne, M. Valkama, Feasibility Study of IEEE 802.11ah Radio Technology for IoT and M2M Use Cases, in: Globecom Workshops (GC Wkshps), IEEE, 1687-1692, 2012.

[17] D. Chen, P. K. Varshney, QoS Support in Wireless Sensor Networks: A Survey., in: International Conference on Wireless Networks, vol. 13244, 227-233, 2004.

[18] G. C. Madueo, . Stefanovi, P. Popovski, Reliable and Efficient Access for Alarm-Initiated and Regular M2M Traffic in IEEE 802.11ah Systems, IEEE Internet of Things Journal 3 (5) (2016) 673-682, ISSN 2327-4662, doi:10.1109/JIOT.2015.2496418.

[19] T. Instruments, CC1100 Datasheet, http://www.ti.com/lit/ds/symlink/cc1100.pdf, [Online; accessed 23-May-2014], 2017.

[20] M. Park, IEEE 802.11 ah: sub-1-GHz license-exempt operation for the internet of things, Communications Magazine, IEEE 53 (9) (2015) 145-151.

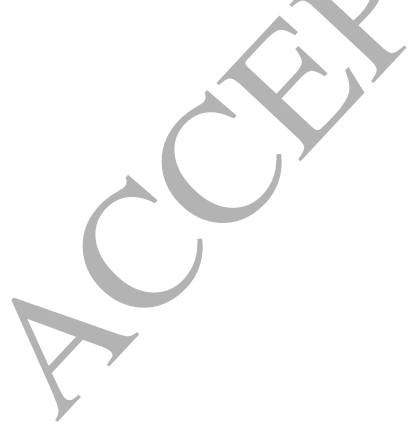




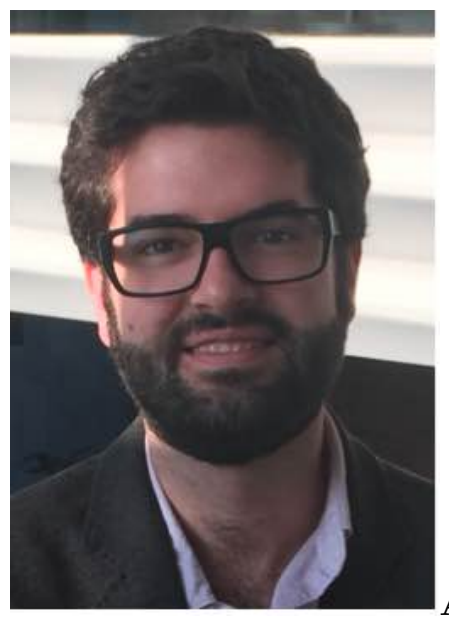

Albert Bel (albert.bel@upf.edu) received his BSc degree in telecommunications engineering (2007), the MSc degree in micro and nanotechnologies (2008), and a PhD in telecommunications and systems engineering (2012) from the Universitat Autonoma de Barcelona. In 2013 he joined the Network Technologies and Strategies (NeTS) group at the Universitat Pompeu Fabra, where he has been involved in different international and national research projects. His research interests are in the area of wireless sensor networks, mostly focused on the design of localization and tracking algorithms and medium access control protocols.

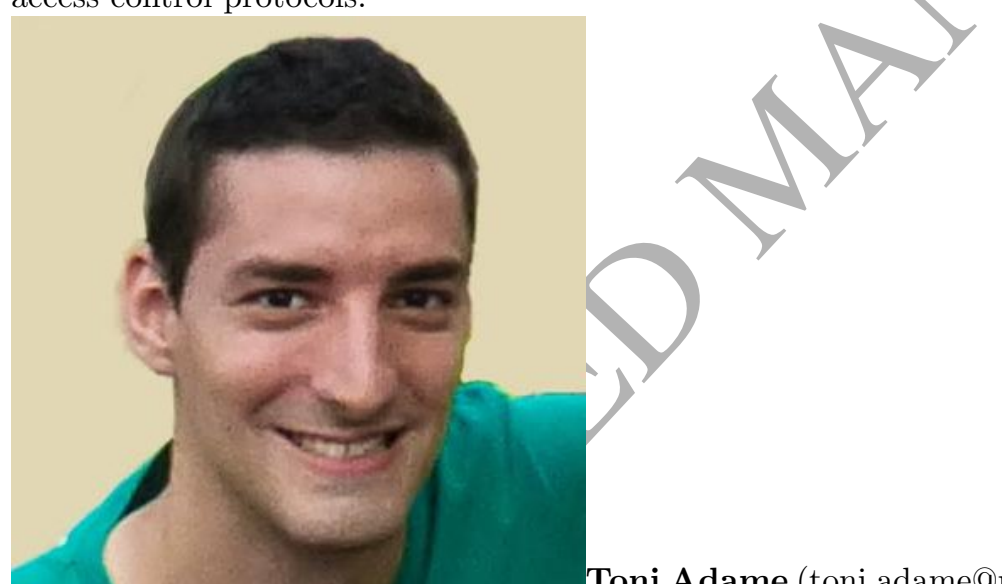

Toni Adame (toni.adame@upf.edu) received his MSc degree in telecommunications engineering from the Universitat Politecnica de Catalunya (UPC) in 2009. After several years working as an IT presales consultant, he joined the Network Technologies and Strategies (NeTS) group in 2013. His research interests are in the area of multiple access communications are Wireless Sensor Networks (WSN), Medium Access Control (MAC) protocols, and power-saving mechanisms. He is currently involved in several national and European research projects related to Wireless Sensor Networks (WSN). 
Boris Bellalta (boris.bellalta@upf.edu) is an Associate Professor in the Department of Information and Communication Technologies (DTIC) at Universitat Pompeu Fabra (UPF). He obtained his degree in Telecommunications Engineering from Universitat Politecnica de Catalunya (UPC) in 2002 and the $\mathrm{PhD}$ in Information and Communication Technologies from UPF in 2007. His research interests are in the area of wireless networks, with emphasis on the design and performance evaluation of new architectures and protocols. The results from his research have been published in more than 100 international journal and conference papers. He is currently involved in several international and national research projects, including the coordination of the ENTOMATIC FP7 collaborative project. At UPF he is giving several courses on networking, queueing theory and wireless networks. He is co-designer and coordinator of the interuniversity (UPF and UPC) masters degree in Wireless Communications. 